\title{
Molecular Biology in Pediatric High-Grade Glioma: Impact on Prognosis and Treatment
}

\author{
Daniela Rizzo, ${ }^{1}$ Antonio Ruggiero, ${ }^{1}$ Maurizio Martini, ${ }^{2}$ \\ Valentina Rizzo, ${ }^{1}$ Palma Maurizi, ${ }^{1}$ and Riccardo Riccardi ${ }^{1}$ \\ ${ }^{1}$ Department of Pediatric Oncology, A. Gemelli Hospital, Catholic University of Rome, \\ Largo A Gemelli, 1, 00168 Rome, Italy \\ ${ }^{2}$ Anatomic Pathology, Catholic University, “A. Gemelli” Hospital, 00168 Rome, Italy
}

Correspondence should be addressed to Antonio Ruggiero; ruggiero@rm.unicatt.it

Received 26 August 2014; Accepted 4 November 2014

Academic Editor: Murat Gokden

Copyright (c) 2015 Daniela Rizzo et al. This is an open access article distributed under the Creative Commons Attribution License, which permits unrestricted use, distribution, and reproduction in any medium, provided the original work is properly cited.

\begin{abstract}
High-grade gliomas are the main cause of death in children with brain tumours. Despite recent advances in cancer therapy, their prognosis remains poor and the treatment is still challenging. To date, surgery followed by radiotherapy and temozolomide is the standard therapy. However, increasing knowledge of glioma biology is starting to impact drug development towards targeted therapies. The identification of agents directed against molecular targets aims at going beyond the traditional therapeutic approach in order to develop a personalized therapy and improve the outcome of pediatric high-grade gliomas. In this paper, we critically review the literature regarding the genetic abnormalities implicated in the pathogenesis of pediatric malignant gliomas and the current development of molecularly targeted therapies. In particular, we analyse the impact of molecular biology on the prognosis and treatment of pediatric high-grade glioma, comparing it to that of adult gliomas.
\end{abstract}

\section{Introduction}

Brain tumors are the most common solid tumors affecting childhood and the main cause of cancer-related death in children. Gliomas make up approximately $60 \%$ of all pediatric brain tumors and about half of these are considered highgrade malignancies. In particular, pediatric glioblastoma (GBM, grade IV WHO) accounts for $15 \%$ of all pediatric brain tumours [1]. Despite efforts to improve treatment, children with high-grade glioma (HGG) still have a dismal outcome with a 5-year survival of less than $20 \%$ [2].

Surgery followed by radiotherapy (RT) is the standard treatment of patients with HGG. Surgery has prognostic significance in patients with near total resection and at present it is the strongest indicator of prognosis in pediatric HGG. Focal RT is used as first-line therapy except in children under 3 years. Recently, the combination of RT and temozolomide (TMZ) followed by adjuvant TMZ showed a superior outcome compared to RT in the treatment of adults with newly diagnosed GBM [3]. However, several studies on the use of TMZ in the treatment of children with HGG were performed with disappointing results (Table 1) [4-9].

Recognizing the limitations of current standard therapy for HGG, over the past few years substantial advances have been made in molecular biology in identifying new therapeutic approaches. Molecular biological investigations have confirmed that the transformed phenotype of HGG is highly complex and is the result of the dysfunction of a variety of interrelated regulatory pathways (Figure 1) [10]. HGG displays complex chromosomal and genetic alterations leading to the inactivation of various tumor suppressor genes, as well as aberrant activation of protooncogenes. The molecular profiling of HGG in adults has been studied intensively because of its relatively high incidence in this population. Conversely, relatively few studies have been performed on pediatric HGG due to the difficulty in obtaining a large enough series of patient samples. Significant differences exist between adult and pediatric HGG, suggesting that 
TABLE 1: Summary of the main clinical trials in pediatric HGG.

\begin{tabular}{|c|c|c|c|c|c|}
\hline $\begin{array}{l}\text { Agent and mechanism of } \\
\text { action }\end{array}$ & Treatment & Study design & Status at diagnosis & Outcome & Reference \\
\hline \multirow{4}{*}{ Alkylating agent (TMZ) } & $\mathrm{RT}+\mathrm{TMZ}$ & Phase II & $\begin{array}{l}\text { Newly diagnosed } \\
\text { HGG }\end{array}$ & $\begin{array}{l}\text { EFS3y: } 11 \pm 3 \% \\
\text { OS3y: } 22 \pm 5 \%\end{array}$ & {$[4]$} \\
\hline & TMZ & Phase II & $\begin{array}{c}\text { Relapsed or } \\
\text { progressive HGG }\end{array}$ & $\begin{array}{l}\text { mOS } 4.7 \text { months } \\
\text { response rate: } 12 \%\end{array}$ & {$[5]$} \\
\hline & $\mathrm{RT}+\mathrm{TMZ}$ & Phase II & $\begin{array}{c}\text { Newly diagnosed } \\
\text { HGG }\end{array}$ & $\begin{array}{l}\text { PFS1y: } 43 \% \pm 9 \% \\
\text { PFS2y: } 11 \% \pm 5 \% \\
\text { OS1y: } 63 \% \pm 8 \% \\
\text { OS2y: } 21 \% \pm 7 \%\end{array}$ & {$[6]$} \\
\hline & TMZ & Phase II & $\begin{array}{c}\text { Relapsed or } \\
\text { progressive HGG }\end{array}$ & $\begin{array}{l}\text { mPFS: } 3 \text { months } \\
\text { PFS6m: } 33 \% \\
\text { mOS: } 4 \text { months } \\
\text { OS6m: } 37.5 \%\end{array}$ & {$[7]$} \\
\hline \multicolumn{6}{|l|}{$\begin{array}{l}\text { Receptor tyrosine kinase } \\
\text { inhibitors }\end{array}$} \\
\hline $\begin{array}{l}\text { EGFR inhibitor } \\
\text { (erlotinib) }\end{array}$ & $\mathrm{RT}+$ erlotinib & Phase I & $\begin{array}{c}\text { Newly diagnosed } \\
\text { HGG }\end{array}$ & $\begin{array}{c}\text { PFS1y: } 56 \% \pm 10 \% \\
\text { PFS2y: } 35 \% \pm 12 \% \\
\text { OS1y: } 78 \% \pm 9 \% \\
\text { OS2y: } 48 \% \pm 12 \%\end{array}$ & {$[31]$} \\
\hline \multirow[t]{2}{*}{$\begin{array}{l}\text { PDGFR inhibitor } \\
\text { (imatinib) }\end{array}$} & Erlotinib & Phase I & $\begin{array}{c}\text { Relapsed or } \\
\text { progressive HGG }\end{array}$ & $\begin{array}{l}\text { mPFS: } 1.5 \text { months } \\
\text { mOS: } 4.1 \text { months } \\
\text { SD: } 28 \%\end{array}$ & {$[33]$} \\
\hline & Imatinib & Phase I & $\begin{array}{c}\text { Relapsed or } \\
\text { progressive HGG }\end{array}$ & $\begin{array}{c}\text { EFS6m: } 17.9 \% \pm 6.6 \% \\
\text { EFS12m: } 0 \%\end{array}$ & {$[51]$} \\
\hline \multirow{3}{*}{$\begin{array}{l}\text { Antiangiogenic agent } \\
(\mathrm{BVZ})\end{array}$} & $\mathrm{BVZ}+$ irinotecan & Phase II & $\begin{array}{c}\text { Relapsed or } \\
\text { progressive HGG }\end{array}$ & $\begin{array}{l}\text { mPFS: } 2.25 \text { months } \\
\text { mOS: } 6.25 \text { months } \\
\text { SD: } 33.3 \%\end{array}$ & {$[65]$} \\
\hline & $\mathrm{BVZ}+$ irinotecan & Phase II & $\begin{array}{c}\text { Relapsed or } \\
\text { progressive HGG }\end{array}$ & $\begin{array}{l}\text { mPFS: } 4,2 \text { months } \\
\text { PFS6m: } 41.8 \%\end{array}$ & {$[64]$} \\
\hline & $\begin{array}{c}\text { BVZ + irinotecan }+ \\
\text { TMZ } \\
\text { (6 patients) } \\
\text { BVZ + irinotecan } \\
\text { (1 patient) } \\
\text { BVZ + CCNU } \\
\text { (1 patient }) \\
\end{array}$ & Phase II & $\begin{array}{c}\text { Relapsed or } \\
\text { progressive HGG }\end{array}$ & $\begin{array}{l}\text { mPFS: } 15 \text { weeks } \\
\text { mOS: } 30 \text { weeks }\end{array}$ & {$[66]$} \\
\hline
\end{tabular}

TMZ: temozolomide; mOS: median overall survival; OS1y: overall survival at 1 year; OS2y: overall survival at 2 years; OS3y: overall survival at 3 years; $\mathrm{mPFS}$ : median progression-free survival; PFS1y: progression-free survival at 1 year; PFS2y: progression-free survival at 2 years; SD: stable disease; EFS6m: event-free survival at 6 months; EFS12m: event-free survival at 12 months; EFS3y: event-free survival at 3 years; RT: radiotherapy; CT: chemotherapy; BVZ: bevacizumab; GBM: glioblastoma multiforme.

the pathways and mechanisms of malignant gliomagenesis are molecularly distinct $[11,12]$. In this review the genetic abnormalities known to be implicated in the pathogenesis of pediatric HGG are described, underlining their prognostic and predictive role and their main impact on the treatment, including the results achieved with new therapeutic agents directed against rational molecular targets (Table 1).

\section{O6-Methylguanine-DNA Methyltransferase (MGMT)}

The antitumor activity of TMZ is due to DNA methylation and O6-methylguanine-DNA methyltransferase (MGMT) is one of the suggested mechanisms of chemoresistance [13]. Cytotoxicity of TMZ is initiated by the methylation of the $\mathrm{O} 6$ 


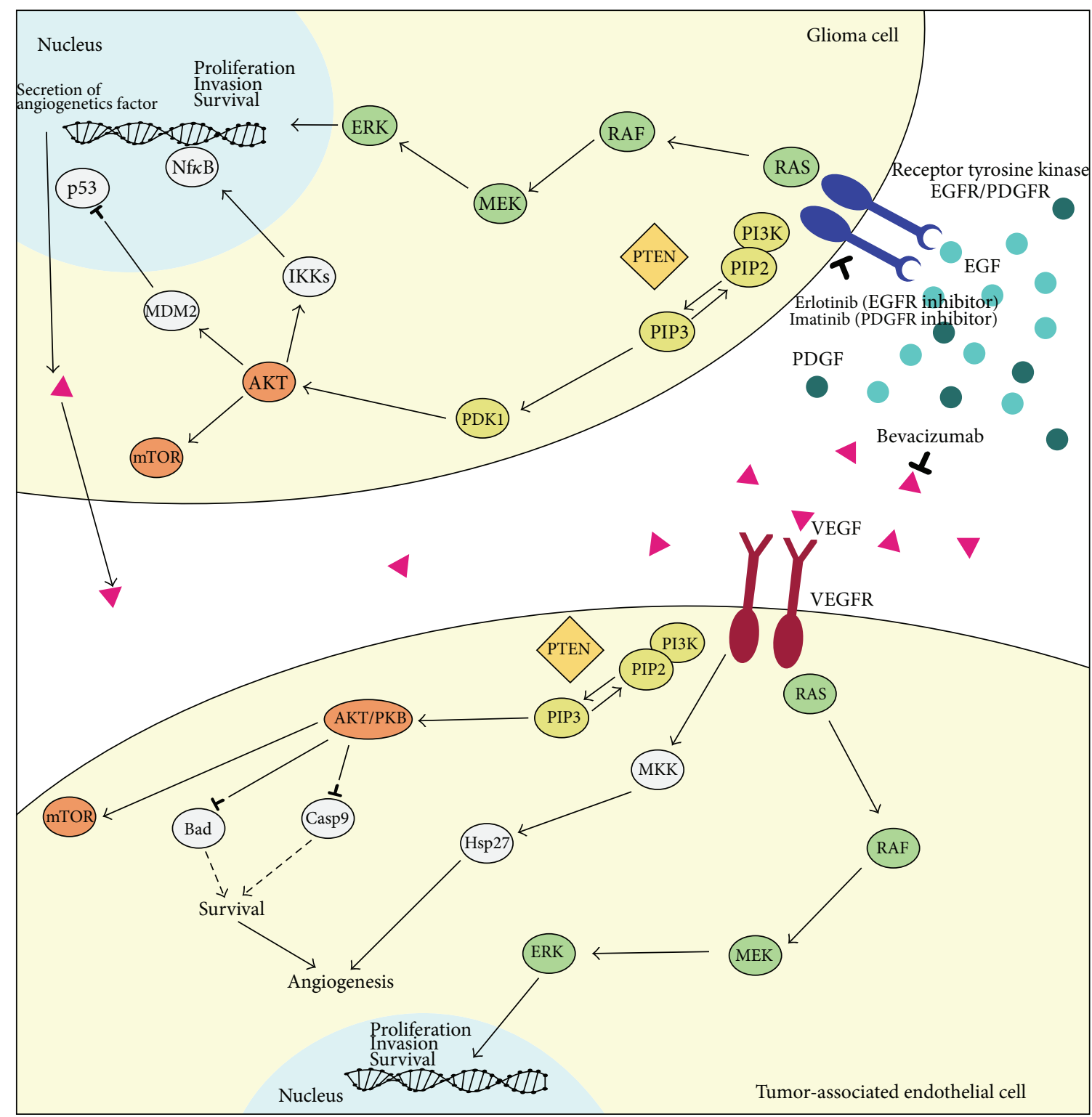

FIGURE 1: Molecularly targeted therapy for pediatric malignant glioma.

position of guanine which causes mispairing of O6-methylguanine with thymine. The futile repair of this base mismatch by the mismatch repair system generates single- and doublestrand DNA breaks that activate cell death. MGMT prevents this process by transferring methylating groups from the $\mathrm{O} 6$ position of guanine to one of its internal cysteine residues, neutralizing the cytotoxic effect of alkylating agents. Therefore elevated MGMT activity is associated with enhanced resistance to alkylating agents.

Stupp et al. showed that MGMT promoter methylation was associated with loss of MGMT expression and diminished DNA-repair activity. Therefore, MGMT promoter methylation represents an independent favorable prognostic factor for GBM patients and a predictive marker of benefit from alkylating agent therapy in GBM [3]. From literature, the percentage of the methylated MGMT promoter of adults ranges from $24 \%$ to $68 \%$, averaging about $40 \%$ [14-16].

Most studies on MGMT methylation in HGG have been conducted in adults, probably due to the relatively rare incidence in children. Only few studies in pediatric populations have been reported [17-20], showing a similar methylation status in children and adults and significant correlation between the methylation status and clinical outcome. In particular, a retrospective study of 10 pediatric patients with GBM showed MGMT promoter methylation in 4 of 10 patients and revealed a significant association between MGMT promoter methylation and prolonged survival $(P=$ 0.01 ) [18]. The average survival time for patients with methylated MGMT was 13.7 months as compared to 2.5 months for the patients with unmethylated $M G M T$ promoter $(P=0.01)$. 
Moreover, the patients receiving TMZ that had the methylated MGMT gene promoter responded better to treatment $(P=0.01)[18]$. In a further study DNA methylation was also associated with increased median event-free survival (EFS) and overall survival (OS) in children with relapsed HGG (5.5 months versus 0.9 months, $P=0.015$ ) [21].

Unlike previous studies, recently Lee et al. showed that the incidence of methylation of the MGMT promoter in pediatric GBM is rare [22]. In this study the methylation status was assessed using two methods: conventional methylationspecific polymerase chain reaction and, for the first time in pediatric patients, methylation-specific multiplex ligationdependent probe amplification. Both methods showed a surprisingly low proportion of methylated samples $(6 \%$ and $16 \%$, resp.). Moreover, there was no difference between the methylated and unmethylated groups in either progressionfree survival (PFS) or OS. Based on limitations of this study, such as small number of patients, heterogeneity in terms of adjuvant treatment modality, location of tumor, and extent of resection, the results should be taken with caution and a multicenter collaborative trial with a larger number of patients should be performed.

\section{Epidermal Growth Factor Receptor (EGFR)}

Epidermal growth factor receptor (EGFR) is a membraneanchored protein tyrosine kinase that, when phosphorylated, activates a variety of downstream effector molecules regulating cell proliferation and differentiation (Figure 1).

Aberrant cell signaling via the EGFR family has been implicated in the development of several human cancers, including brain tumors. Moreover, recent research has shown a pivotal relationship between EGFR overexpression or EGFR amplification and disease progression, poor survival, resistance to chemotherapy, and poor response [23]. EGFR amplification and EGFR overexpression affect $30-50 \%$ of adult GBM [24]. In pediatric HGGs available data suggest that EGFR amplification occurs with low frequency, averaging about 3\% [11, 25], although in a recent study Bax et al. found a greater prevalence of EGFR gene amplification and EGFRvIII mutation in pediatric HGG than had previously been recognized [26]. EGFR $v I I I$ is the most common mutant of EGFR gene reported in GBM. The ability of this variant to "switch on" cell signaling without ligand stimulation, even though it does not dimerize, plays an important role in cancer pathogenesis.

Erlotinib, a small-molecule EGFR inhibitor, has been shown to inhibit EGFRvIII by blocking constitutive EGFRvIII kinase activity and the growth of EGFRvIII transformed cells $[27,28]$. However, several clinical trials have demonstrated a limited activity of erlotinib in the treatment of adults and children with recurrent HGG [29-33]. In particular only a subset of patients treated by EGFR inhibitors showed a response to these agents. Mellinghoff et al. demonstrated that coexpression of EGFRvIII and phosphatase and tensin homolog (PTEN) was associated with better response in patients with HGG treated by EGFR inhibitors [27]. HaasKogan et al. showed that patients with GBM whose tumors expressed high levels of EGFR and low levels of phosphorylated protein kinase $\mathrm{B} / \mathrm{Akt}$ responded better to erlotinib therapy [34]. A recent study indicated that 5 genes within the EGFR signaling pathway (STAT1, FKBP14, RAC1, PTGER4, and $M Y C$ ) may modulate the response of adult GBM to erlotinib [35]. In the study by Bax et al. phosphorylated receptor tyrosine kinase profiling showed a specific activation of platelet-derived growth factor receptor (PDGFR) $\alpha / \beta$ in EGFRvIII-transduced pediatric GBM cells and targeted coinhibition with erlotinib and imatinib, an inhibitor of the tyrosine kinases Bcr-Abl, Kit, and PDGFR, could lead to enhanced efficacy [26]. This suggests that the erlotinibassociated signaling pathway is a complex one and needs to be taken into account in future trials.

\section{Platelet-Derived Growth Factor Receptor (PDGFR)}

Platelet-derived growth factor (PDGF) is a major regulator of angiogenesis [36] and is involved in the regulation of proliferation, neuronal differentiation, and motility of cells within the nervous system [37]. PDGF encompasses a family of ligands ( $\mathrm{AA}, \mathrm{AB}, \mathrm{BB}, \mathrm{CC}$, and $\mathrm{DD}$ ) that bind to a pair of receptors (PDGFR $\alpha$ and PDGFR $\beta$ ) [38]. Concurrent expression of one or more of these ligands and their receptors has been observed in a high percentage of malignant gliomas $[38,39]$, thus allowing autocrine and paracrine stimulation $[38,40]$. In particular, PDGFRA is the most frequent target of focal amplification in pediatric HGGs arising within and outside the brainstem [11,41-44] and somatic mutations of PDGFRA have been recently reported in pediatric HGGs [45, 46]. In contrast, EGFR is the predominant receptor tyrosine kinase targeted by both amplification and mutation in adult GBM [25, 47].

Amplification of wild-type PDGFRA occurred more frequently in tumors within the brainstem (26\% DIPG versus $11 \%$ nonbrainstem HGG, $P=0.04$ ), whereas PDGFRA sequence mutations were more common in pediatric HGG arising outside the brainstem, although this was not statistically significant (14\% nonbrainstem HGG versus 5\% DIPG, $P=0.14[11,41])$.

Imatinib (Gleevec) is a molecular targeted drug which selectively inhibits several receptor tyrosine kinases, including PDGFR (Figure 1). Imatinib was evaluated in several clinical trials, with the drug showing limited activity in the monotherapy of adult GBM [48, 49]. Conversely, a substantial antitumor activity of imatinib in combination with hydroxyurea was described in further open-label trials [50]. Nevertheless, the results have been disappointing in pediatric HGG [51] (Table 1) and further trials looking at combination therapy are required. The main reason for the limited activity of imatinib may be that inhibition of PDGFR alone is insufficient to prevent growth of malignant gliomas. Signaling through the Ras-mitogen-activated protein kinase and Akt pathways as a result of EGFR amplification and mutation and deletion of $P T E N$, respectively, may result in tumor growth even in the presence of PDGFR inhibition. 


\section{Phosphatase and Tensin Homolog (PTEN)/Akt}

The phosphatidylinositol 3-kinase (PI3K) pathway is involved in several important cellular functions, including growth control, survival, and migration. Following its activation by growth factor receptors, including EGFR, EGFRvIII, and PDGFR, PI3K catalyzes the addition of a phosphate to phosphatidylinositol-4,5,-bisphosphate to form phosphatidylinositol-3,4,5,-triphosphate (PIP3), which initiates many of its tumorigenic activities via Akt (Figure 1). Akt is recruited to the plasma membrane by PI3K mediated formation of PIP3, leading to Akt phosphorylation at thr308 and Ser473 (via phosphoinositide-dependent kinase-1 and phosphoinositide-dependent kinase-2, resp.). Akt phosphorylation at these 2 sites activates its kinase function, leading to downstream signaling promoting proliferation and inhibiting apoptosis.

The PTEN gene is an important regulator of the PI3K pathway (Figure 1). It is a tumor suppressor gene which encodes a phosphatase catalyzing the dephosphorylation of PIP3, thus inhibiting activation of the Akt pathway. When PTEN is altered, the Akt pathway can become constitutively active. Elevated Akt levels have been associated with loss of PTEN in many GBMs. Moreover, in glial tumors, PTEN mutation frequency increases with increasing tumor grade and is associated with poorer outcome [52, 53].

Although PTEN mutations are found in approximately $50 \%$ of adult HGG, predominantly GBM, a relatively small rate of mutation was found in childhood gliomas [25, 53, 54]. However, in a recent study Pollack et al. observed that activation of Akt is a common finding in pediatric malignant gliomas. In particular, 42 (79\%) of the 53 evaluable tumors showed overexpression of activated Akt which was associated with a poor prognosis: 1-year EFS was 59\% for patients with Akt overexpression and $91 \%$ for those with no overexpression ( $P=0.16)$; 1 -year OS was $78 \%$ and $100 \%$, respectively $(P=0.06)$ [55]. These data were confirmed in a further series of 32 pediatric GBM samples showing an association between Ras/Akt activation and poor survival [1]. In view of the frequency of Akt activation, the evaluation of molecularly targeted therapies that inhibit this pathway warrants consideration as far as these tumors are concerned.

\section{Vascular Endothelial Growth Factor (VEGF)}

HGGs are some of the most vascularised human tumours and the role of angiogenesis in malignant gliomas has been a very active area of research, with significant impact on the development of targeted therapy [56]. Vascular endothelial growth factor (VEGF) is an important regulator of angiogenesis which is strongly expressed in HGG (Figure 1). The degree of both vasculature density and VEGF expression is associated with the malignancy and aggressiveness of these tumors, as well as with outcomes such as clinical recurrence and survival [57-59]. Recently, data from clinical trials have established antiangiogenic therapy with VEGF targeted agents, with or without cytotoxic chemotherapy, as an active treatment option for patients with recurrent HGG who have failed previous TMZ therapy. Bevacizumab (BVZ) recently received Food and Drug Administration approval as a single agent for the treatment of patients with progressive GBM.

BVZ has been administered with irinotecan, a topoisomerase 1 inhibitor, in patients with recurrent $\mathrm{HGG}$, and this combination has shown activity. BVZ in combination with irinotecan has now been reported in several trials to improve the outcome of recurrent malignant glioma. Both complete and partial responses, as well as disease stabilisation, have been described [60-62]. Moreover, recently, BVZ in combination with standard upfront treatment showed encouraging results [63].

Despite clear evidence of BVZ activity in recurrent HGG, not all patients respond to treatment, and no biomarkers for patients responsive to antiangiogenic therapies have been identified. Moreover, the activity of BVZ was found to be lower in pediatric gliomas, suggesting that genetic differences in pediatric gliomas might account for this difference.

A phase II pediatric brain tumor consortium study of a combination of BVZ and irinotecan was performed in children with recurrent malignant glioma by Gururangan et al., which reported a median PFS of 4.2 months and no sustained responses among the 15 children studied [64]. Similar results were reported in 12 children with recurrent or progressive HGG. Treatment tolerance and toxicity were comparable to adult HGG patients; however, the radiological response rate, response duration, and survival appeared inferior in pediatric patients [65]. Moreover bevacizumab was also investigated in combination with different drugs, such as irinotecan, CCNU, and TMZ, in children with recurrent or progressive WHO grades 3-4 gliomas, and although the combination was well tolerated, it lacked efficacy, with no sustained responses observed [66].

\section{TP53}

The TP53 pathway is an important mechanism controlling the cell cycle (Figure 1). Activation of the tumor suppressor p53 by stress signals triggers different cellular programs such as cell cycle arrest, apoptosis, differentiation, DNA repair, autophagy, and senescence through complex network and signaling pathways. Childhood multi-institutional studies have confirmed that p53 overexpression and mutation appear to vary with age, being expressed more strongly in older versus younger children $[67,68]$. In the Children's Cancer Group Study, the largest cohort of childhood HGGs analyzed to date, TP53 mutations were observed in only 2/17 tumors $(11.8 \%)$ from children $<3$ years of age at diagnosis versus $24 / 60$ tumors (40\%) from older children, a difference that is statistically significant $(P=0.04)$.

Moreover, a significant association has also been found between overexpression of p53, even in the absence of TP53 mutations, and HGG outcome in children. The rate of PFS at 5 years was $44 \%$ in the group of patients whose tumors had low levels of expression of p53 and only 17\% in the group of patients whose tumors had overexpression of p53 $(P<0.001)$ [68]. Moreover, overexpression of the $\mathrm{p} 53$ protein increases with tumor grade: one-fourth of analyzed AAs and half of GBMs overexpressed this protein. 


\section{Histone H3.3}

Histones are eukaryotic nuclear proteins that play an important role in the regulation of DNA replication, transcription, and storage by changing the nucleosome structure depending on their post translational modifications. H3.3 is a replacement histone subclass that is encoded by 2 distinct genes, H3.3A (H3F3A) and H3.3B [69]. H3.3 is the major histone to be loaded on chromatin during brain development. This histone variant is known to modulate specific chromatin changes and gene expression profiles and to be associated with active chromatin and translation. Recent studies investigated the cancer genome of pediatric diffuse glioma $[46,70]$. Exon sequencing identified mutations in histone $\mathrm{H} 3.3$ at either aminoacid 27, resulting in replacement of lysine by methionine (K27M), or at aminoacid 34, resulting in replacement of glycine by valine or arginine (G34V/R), as molecular drivers of a subgroup of pediatric and young adult GBMs and pediatric diffuse intrinsic pontine gliomas. In particular one-fourth of pediatric astrocytomas showed somatic mutations in $H 3 F 3 A$ gene [46, 70-73]. G34V/R-H3.3 was mainly seen in older patients (median age 20 years) and almost exclusively in hemispheric HGGs [53]. Notably, in GBM patients G34 mutant also showed a trend toward a better OS than wild-type tumor patients, with marginal statistical significance $(P=0.05)$ [55]. In contrast, patients who harbored the K27M-H3.3 mutation tended toward a worse overall survival when compared to patients who were wild-type for H3.3. Moreover, H3F3A K27 mutation appeared to be exclusive to pediatric high-grade gliomas (median age 11 years) $[46,73]$ and it was prevalent in diffuse intrinsic pontine glioma $[70,72]$. Therefore this mutation defines clinically and biologically distinct subgroup and it is suitable as a molecular marker for pediatric diffuse high-grade astrocytomas with a future impact on therapeutic trial design.

\section{Alpha-Thalassemia/Mental-Retardation Syndrome X-Linked (ATRX)}

The ATRX (alpha-thalassemia/mental-retardation syndrome $\mathrm{X}$-linked) gene is located on chromosome Xq21.1 and encodes a subunit of a chromatin remodelling complex required for H3.3 incorporation at pericentric heterochromatin and telomeres [74]. Mutations that inactivate ATRX gene are common in human pancreatic neuroendocrine tumors and central nervous system tumors [70-75]. Loss of ATRX function impairs the heterochromatic state of the telomeres, perhaps because of reduced incorporation of chromatin onto H3.3 histones [75]. This leads to telomere destabilization, which results in a telomerase-independent telomere maintenance mechanism called alternative lengthening of telomeres (ALT).

In 2011, mutations in the ATRX gene were described for the first time in a small fraction of adult and pediatric GBM, as well as oligodendrogliomas, and a significant correlation with ALT was demonstrated [75]. Recently, mutations and loss of ATRX have been reported in one-third of pediatric
GBMs [46] and 7\% of adult GBMs [75]. In gliomas, ATRX mutation has been associated with a better prognosis in anaplastic gliomas [76].

Khuong-Quang et al. showed that the presence of ATRX mutation significantly overlapped with TP53 mutations in GBM $(P=0.01)$ regardless of the location within the brain and with $\mathrm{G} 34 \mathrm{~V} / \mathrm{R}$ mutants in supratentorial GBM $(P<$ $0.0001)$. Moreover ATRX mutations were infrequent in DIPG and mainly occurred in older children $(P<0.0001)$. The low incidence of this mutation in DIPG could be an agerelated phenomenon as the mean age of DIPG cohort was 7 versus 12 years for the supratentorial GBM patient cohort [72]. The requirement for ATRX mutations in GBM may thus be due to tumor location and/or the age of the patient. This is potentially indicative of a different cell of origin or age-related plasticity of the tumor.

\section{Isocitrate Dehydrogenase (IDH)}

Recently a high frequency of mutations of the isocitrate dehydrogenase (IDH1 and IDH2) genes, which encode the IDH enzymes, was detected in adult secondary GBM (85\%). These alterations inhibit the normal function of the IDH enzyme in converting isocitrate to $\alpha$-ketoglutarate [77] and instead drive the conversion of $\alpha$-ketoglutarate to $\mathrm{R}(-)$ 2-hydroxyglutarate [35], a metabolite that may contribute to tumor development. IDH mutations likely represent an early step in tumorigenesis because such alterations are also observed commonly in preexisting lower grade lesions [78].

Such alterations are uncommon in pediatric population, highlighting molecular differences with adult secondary GBM.

The series of Balss et al. [79] included 14 pediatric GBMs and only one case had an $I D H 1$ mutation. In 2 recent studies performed on pediatric malignant gliomas IDH1 mutations were not detected in any case $[80,81]$. Similarly, in a study of 1010 diffuse gliomas that included a small subgroup of children, Hartmann et al. [82] noted that IDH1 mutations were rare in the pediatric subset, and IDH2 mutations were absent, although the frequency of mutations as a function of age and histology was not provided.

Based on the molecular similarities that have been noted between primary pediatric malignant gliomas that arise in older children and secondary malignant gliomas that occur in adults, recently Pollack et al. examined the frequency of $I D H$ mutations in a cohort of 43 HGG patients aged 3-21 years at the time of diagnosis [83]. IDH1 mutations were noted in 7 of 20 tumors (35\%) from children $\geq 14$ years, but in 0 of 23 (0\%) younger children $(P=0.01)$, suggesting that a subset of such lesions may be comparable on a molecular basis to lesions that arise in young adults. In contrast, such alterations were rare in tumors arising in younger children, supporting the existence of age-related pathways of tumorigenesis in childhood. Moreover IDH-mutated tumors seemed to be associated with a more favorable prognosis than non mutated tumors as in adult patients [80]. 


\section{Conclusion}

HGGs are highly heterogeneous and aggressive brain tumours, requiring a multidisciplinary approach. Recently, molecular research has significantly improved our understanding of glioma pathogenesis and identified key proteins that regulate normal biological processes and cellular pathways associated with pediatric HGG. The identification of agents directed against molecular targets aims at going beyond the traditional therapeutic approach in order to develop a personalized therapy and improve the outcome of pediatric HGG.

Results from adult clinical trials cannot simply be extrapolated to children due to crucial molecular differences between adult and pediatric HGG, drawing attention to the need for exclusively pediatric clinical studies. PDGFRA is the most frequent target of focal amplification in pediatric HGGs; in contrast, EGFR is the predominant receptor tyrosine kinase targeted in adult GBM. No IDH1 mutations have been found in pediatric tumors, highlighting molecular differences with adult secondary GBM. Activity of bevacizumab is lower in pediatric gliomas than that observed in adult HGG. Recently a central role of $A T R X$ and H3F3A K27 mutations in pediatric $H H G$ s has been described.

Although targeted agents offer great promise, to date the overall response rate has been disappointing, with differences in response not only between children and adults but also within each population. These observations demonstrate that distinct biological profiles can be identified in different subsets of patients; therefore individual targeted therapy based on molecular biology should be investigated in order to define an optimal treatment strategy for each patient.

\section{Conflict of Interests}

The authors declare that there is no conflict of interests regarding the publication of this paper.

\section{References}

[1] D. Faury, A. Nantel, S. E. Dunn et al., "Molecular profiling identifies prognostic subgroups of pediatric glioblastoma and shows increased YB-1 expression in tumors," Journal of Clinical Oncology, vol. 25, no. 10, pp. 1196-1208, 2007.

[2] A. Broniscer and A. Gajjar, "Supratentorial high-grade astrocytoma and diffuse brainstem glioma: two challenges for the pediatric oncologist," Oncologist, vol. 9, no. 2, pp. 197-206, 2004.

[3] R. Stupp, W. P. Mason, M. J. van den Bent et al., "Radiotherapy plus concomitant and adjuvant temozolomide for glioblastoma," The New England Journal of Medicine, vol. 352, no. 10, pp. 987-996, 2005.

[4] K. J. Cohen, I. F. Pollack, T. Zhou et al., "Temozolomide in the treatment of high-grade gliomas in children: a report from the Children's Oncology Group," Neuro-Oncology, vol. 13, no. 3, pp. 317-323, 2011.

[5] L. S. Lashford, P. Thiesse, A. Jouvet et al., "Temozolomide in malignant gliomas of childhood: A United Kingdom Children's Cancer Study Group and French Society for Pediatric Oncology Intergroup Study," Journal of Clinical Oncology, vol. 20, no. 24, pp. 4684-4691, 2002.
[6] A. Broniscer, M. Chintagumpala, M. Fouladi et al., "Temozolomide after radiotherapy for newly diagnosed high-grade glioma and unfavorable low-grade glioma in children," Journal of Neuro-Oncology, vol. 76, no. 3, pp. 313-319, 2006.

[7] A. Ruggiero, G. Cefalo, M. L. Garré et al., "Phase II trial of temozolomide in children with recurrent high-grade glioma," Journal of Neuro-Oncology, vol. 77, no. 1, pp. 89-94, 2006.

[8] H. S. Nicholson, C. S. Kretschmar, M. Krailo et al., "Phase 2 study of temozolomide in children and adolescents with recurrent central nervous system tumors: a report from the Children's Oncology Group," Cancer, vol. 110, no. 7, pp. 15421550, 2007.

[9] K. J. Cohen, R. L. Heideman, T. Zhou et al., "Temozolomide in the treatment of children with newly diagnosed diffuse intrinsic pontine gliomas: a report from the Children's Oncology Group," Neuro-Oncology, vol. 13, no. 4, pp. 410-416, 2011.

[10] E. A. Maher, F. B. Furnari, R. M. Bachoo et al., "Malignant glioma: genetics and biology of a grave matter," Genes \& Development, vol. 15, no. 11, pp. 1311-1333, 2001.

[11] B. S. Paugh, C. Qu, C. Jones et al., "Integrated molecular genetic profiling of pediatric high-grade gliomas reveals key differences with the adult disease," Journal of Clinical Oncology, vol. 28, no. 18, pp. 3061-3068, 2010.

[12] T. J. MacDonald, D. Aguilera, and C. M. Kramm, "Treatment of high-grade glioma in children and adolescents," NeuroOncology, vol. 13, no. 10, pp. 1049-1058, 2011.

[13] R. Stupp, M. Gander, S. Leyvraz, and E. Newlands, "Current and future developments in the use of temozolomide for the treatment of brain tumours," The Lancet Oncology, vol. 2, no. 9, pp. 552-560, 2001.

[14] M. Esteller, J. Garcia-Foncillas, E. Andion et al., "Inactivation of the DNA-repair gene MGMT and the clinical response of gliomas to alkylating agents," The New England Journal of Medicine, vol. 343, no. 19, pp. 1350-1354, 2000.

[15] M. E. Hegi, A.-C. Diserens, T. Gorlia et al., "MGMT gene silencing and benefit from temozolomide in glioblastoma," The New England Journal of Medicine, vol. 352, no. 10, pp. 997-1003, 2005.

[16] M. Eoli, F. Menghi, M. G. Bruzzone et al., "Methylation of O6-methylguanine DNA methytransferase and loss of heterozygosity on $19 q$ and/or $17 p$ are overlapping features of secondary glioblastomas with prolonged survival," Clinical Cancer Research, vol. 13, no. 9, pp. 2606-2613, 2007.

[17] I. F. Pollack, R. L. Hamilton, R. W. Sobol et al., "O6Methylguanine-DNA methyltransferase expression strongly correlates with outcome in childhood malignant gliomas: results from the CCG-945 cohort," Journal of Clinical Oncology, vol. 24, no. 21, pp. 3431-3437, 2006.

[18] A. M. Donson, S. O. Addo-Yobo, M. H. Handler, L. Gore, and N. K. Foreman, "MGMT promoter methylation correlates with survival benefit and sensitivity to temozolomide in pediatric glioblastoma," Pediatric Blood and Cancer, vol. 48, no. 4, pp. 403-407, 2007.

[19] F. R. Buttarelli, M. Massimino, M. Antonelli et al., "Evaluation status and prognostic significance of O6-methylguanine-DNA methyltransferase (MGMT) promoter methylation in pediatric high grade gliomas," Child's Nervous System, vol. 26, no. 8, pp. 1051-1056, 2010.

[20] A. Srivastava, A. Jain, P. Jha et al., "MGMT gene promoter methylation in pediatric glioblastomas," Child's Nervous System, vol. 26, no. 11, pp. 1613-1618, 2010. 
[21] S. Schlosser, S. Wagner, J. M. Hlisch et al., "MGMT as a potential stratification marker in relapsed high-grade glioma of children: the HIT-GBM experience," Pediatric Blood \& Cancer, vol. 54, no. 2, pp. 228-237, 2010.

[22] J. Y. Lee, C.-K. Park, S.-H. Park, K.-C. Wang, B.-K. Cho, and S.-K. Kim, "MGMT promoter gene methylation in pediatric glioblastoma: analysis using MS-MLPA," Child's Nervous System, vol. 27, no. 11, pp. 1877-1883, 2011.

[23] N. Shinojima, K. Tada, S. Shiraishi et al., "Prognostic value of epidermal growth factor receptor in patients with glioblastoma multiforme," Cancer Research, vol. 63, no. 20, pp. 6962-6970, 2003.

[24] T. A. Libermann, H. R. Nusbaum, N. Razon et al., "Amplification, enhanced expression and possible rearrangement of EGF receptor gene in primary human brain tumours of glial origin," Nature, vol. 313, no. 5998, pp. 144-147, 1985.

[25] I. F. Pollack, R. L. Hamilton, C. D. James et al., "Rarity of PTEN deletions and EGFR amplification in malignant gliomas of childhood: results from the Children's Cancer Group 945 cohort," Journal of Neurosurgery, vol. 105, no. 5, pp. 418-424, 2006.

[26] D. A. Bax, N. Gaspar, S. E. Little et al., "EGFRvIII deletion mutations in pediatric high-grade glioma and response to targeted therapy in pediatric glioma cell lines," Clinical Cancer Research, vol. 15, pp. 5753-5761, 2009.

[27] I. K. Mellinghoff, M. Y. Wang, I. Vivanco et al., "Molecular determinants of the response of glioblastomas to EGFR kinase inhibitors," The New England Journal of Medicine, vol. 353, no. 19, pp. 2012-2024, 2005.

[28] I. K. Mellinghoff, T. F. Cloughesy, and P. S. Mischel, "PTENmediated resistance to epidermal growth factor receptor kinase inhibitors," Clinical Cancer Research, vol. 13, no. 2 I, pp. 378-381, 2007.

[29] M. D. Prados, K. R. Lamborn, S. Chang et al., "Phase 1 study of erlotinib $\mathrm{HCl}$ alone and combined with temozolomide in patients with stable or recurrent malignant glioma," NeuroOncology, vol. 8, no. 1, pp. 67-78, 2006.

[30] W. K. A. Yung, J. J. Vredenburgh, T. F. Cloughesy et al., "Safety and efficacy of erlotinib in first-relapse glioblastoma: a phase II open-label study," Neuro-Oncology, vol. 12, no. 10, pp. 1061-1070, 2010.

[31] A. Broniscer, S. J. Baker, C. F. Stewart et al., "Phase Ii and pharmacokinetic studies of erlotinib administered concurrently with radiotherapy for children, adolescents, and young adults with high-grade glioma," Clinical Cancer Research, vol. 15, no. 2, pp. 701-707, 2009.

[32] R. I. Jakacki, M. Hamilton, R. J. Gilbertson et al., "Pediatric phase I and pharmacokinetic study of erlotinib followed by the combination of erlotinib and temozolomide: a children's oncology group phase I consortium study," Journal of Clinical Oncology, vol. 26, no. 30, pp. 4921-4927, 2008.

[33] B. Geoerger, D. Hargrave, F. Thomas et al., "Innovative therapies for children with cancer pediatric phase I study of erlotinib in brainstem glioma and relapsing/refractory brain tumors," Neuro-Oncology, vol. 13, no. 1, pp. 109-118, 2011.

[34] D. A. Haas-Kogan, M. D. Prados, T. Tihan et al., "Epidermal growth factor receptor, protein kinase $\mathrm{B} / \mathrm{Akt}$, and glioma response to erlotinib," Journal of the National Cancer Institute, vol. 97, no. 12, pp. 880-887, 2005.

[35] M.-E. Halatsch, S. Löw, T. Hielscher, U. Schmidt, A. Unterberg, and V. I. Vougioukas, "Epidermal growth factor receptor pathway gene expressions and biological response of glioblastoma multiforme cell lines to erlotinib," Anticancer Research, vol. 28, no. 6A, pp. 3725-3728, 2008.

[36] D. Wang, H.-J. S. Huang, A. Kazlauskas, and W. K. Cavenee, "Induction of vascular endothelial growth factor expression in endothelial cells by platelet-derived growth factor through the activation of phosphatidylinositol 3-kinase," Cancer Research, vol. 59, no. 7, pp. 1464-1472, 1999.

[37] A. Erlandsson, M. Enarsson, and K. Forsberg-Nilsson, "Immature neurons from CNS stem cells proliferate in response to platelet-derived growth factor," Journal of Neuroscience, vol. 21, no. 10, pp. 3483-3491, 2001.

[38] N. A. Lokker, C. M. Sullivan, S. J. Hollenbach, M. A. Israel, and N. A. Giese, "Platelet-derived growth factor (PDGF) autocrine signaling regulates survival and mitogenic pathways in glioblastoma cells: evidence that the novel PDGF-C and PDGF-D ligands may play a role in the development of brain tumors," Cancer Research, vol. 62, no. 13, pp. 3729-3735, 2002.

[39] M. Maxwell, S. P. Naber, H. J. Wolfe et al., "Coexpression of platelet-derived growth factor (PDGF) and PDGF-receptor genes by primary human astrocytomas may contribute to their development and maintenance," The Journal of Clinical Investigation, vol. 86, no. 1, pp. 131-140, 1990.

[40] F. S. Vassbotn, A. Östman, N. Langeland et al., "Activated platelet-derived growth factor autocrine pathway drives the transformed phenotype of a human glioblastoma cell line," Journal of Cellular Physiology, vol. 158, no. 2, pp. 381-389, 1994.

[41] B. S. Paugh, A. Broniscer, C. Qu et al., "Genome-wide analyses identify recurrent amplifications of receptor tyrosine kinases and cell-cycle regulatory genes in diffuse intrinsic pontine glioma," Journal of Clinical Oncology, vol. 29, no. 30, pp. 39994006, 2011.

[42] S. Puget, C. Philippe, D. A. Bax et al., "Mesenchymal transition and pdgfra amplification/mutation are key distinct oncogenic events in pediatric diffuse intrinsic pontine gliomas," PLoS ONE, vol. 7, no. 2, Article ID e30313, 2012.

[43] M. Zarghooni, U. Bartels, E. Lee et al., "Whole-genome profiling of pediatric diffuse intrinsic pontine gliomas highlights plateletderived growth factor receptor $\alpha$ and poly (ADP-ribose) polymerase as potential therapeutic targets," Journal of Clinical Oncology, vol. 28, no. 8, pp. 1337-1344, 2010.

[44] D. A. Bax, A. Mackay, S. E. Little et al., "A distinct spectrum of copy number aberrations in pediatric high-grade gliomas," Clinical Cancer Research, vol. 16, no. 13, pp. 3368-3377, 2010.

[45] B. S. Paugh, X. Zhu, C. Qu et al., "Novel oncogenic PDGFRA mutations in pediatric high-grade gliomas," Cancer Research, vol. 73, no. 20, pp. 6219-6229, 2013.

[46] J. Schwartzentruber, A. Korshunov, X.-Y. Liu et al., "Driver mutations in histone $\mathrm{H} 3.3$ and chromatin remodelling genes in paediatric glioblastoma," Nature, vol. 482, no. 7384, pp. 226-231, 2012.

[47] F. B. Furnari, T. Fenton, R. M. Bachoo et al., "Malignant astrocytic glioma: genetics, biology, and paths to treatment," Genes and Development, vol. 21, no. 21, pp. 2683-2710, 2007.

[48] E. Raymond, A. A. Brandes, C. Dittrich et al., "Phase II study of imatinib in patients with recurrent gliomas of various histologies: a European organisation for research and treatment of cancer brain tumor group study," Journal of Clinical Oncology, vol. 26, no. 28, pp. 4659-4665, 2008.

[49] P. Y. Wen, W. K. A. Yung, K. R. Lamborn et al., "Phase I/II study of imatinib mesylate for recurrent malignant gliomas: north American brain tumor consortium study 99-08," Clinical Cancer Research, vol. 12, no. 16, pp. 4899-4907, 2006. 
[50] D. A. Reardon, M. J. Egorin, J. A. Quinn et al., "Phase II study of imatinib mesylate plus hydroxyurea in adults with recurrent glioblastoma multiforme," Journal of Clinical Oncology, vol. 23, pp. 9359-9368, 2005.

[51] I. F. Pollack, R. I. Jakacki, S. M. Blaney et al., "Phase I trial of imatinib in children with newly diagnosed brainstem and recurrent malignant gliomas: a Pediatric Brain Tumor Consortium report," Neuro-Oncology, vol. 9, no. 2, pp. 145-160, 2007.

[52] J. S. Smith, I. Tachibana, S. M. Passe et al., "PTEN mutation, EGFR amplification, and outcome in patients with anaplastic astrocytoma and glioblastoma multiforme," Journal of the National Cancer Institute, vol. 93, no. 16, pp. 1246-1256, 2001.

[53] C. Raffel, L. Frederick, J. R. O’Fallon et al., “Analysis of oncogene and tumor suppressor gene alterations in pediatric malignant astrocytomas reveals reduced survival for patients with PTEN mutations," Clinical Cancer Research, vol. 5, no. 12, pp. 40854090, 1999.

[54] J. A. Kraus, J. Felsberg, J. C. Tonn, G. Reifenberger, and T. Pietsch, "Molecular genetic analysis of the TP53, PTEN, CDKN2A, EGFR, CDK4 and MDM2 tumour-associated genes in supratentorial primitive neuroectodermal tumours and glioblastomas of childhood," Neuropathology and Applied Neurobiology, vol. 28, no. 4, pp. 325-333, 2002.

[55] I. F. Pollack, R. L. Hamilton, P. C. Burger et al., "Akt activation is a common event in pediatric malignant gliomas and a potential adverse prognostic marker: a report from the Children's Oncology Group," Journal of Neuro-Oncology, vol. 99, no. 2, pp. 155$163,2010$.

[56] N. Oka, A. Soeda, A. Inagaki et al., "VEGF promotes tumorigenesis and angiogenesis of human glioblastoma stem cells," Biochemical and Biophysical Research Communications, vol. 360, no. 3, pp. 553-559, 2007.

[57] S. P. Leon, R. D. Folkerth, and P. M. Black, "Microvessel density is a prognostic indicator for patients with astroglial brain tumors," Cancer, vol. 77, no. 2, pp. 362-372, 1996.

[58] N. O. Schmidt, M. Westphal, C. Hagel et al., "Levels of vascular endothelial growth factor, hepatocyte growth factor/scatter factor and basic fibroblast growth factor in human gliomas and their relation to angiogenesis," International Journal of Cancer, vol. 84, pp. 10-18, 1999.

[59] Y.-H. Zhou, F. Tan, K. R. Hess, and W. K. A. Yung, "The expression of PAX6, PTEN, vascular endothelial growth factor, and epidermal growth factor receptor in gliomas: relationship to tumor grade and survival," Clinical Cancer Research, vol. 9, no. 9, pp. 3369-3375, 2003.

[60] D. Schiff and B. Purow, "Bevacizumab in combination with irinotecan for patients with recurrent glioblastoma multiforme: commentary," Nature Clinical Practice Oncology, vol. 5, no. 4, pp. 186-187, 2008.

[61] M. C. Chamberlain, "Bevacizumab plus irinotecan in recurrent glioblastoma," Journal of Clinical Oncology, vol. 26, no. 6, pp. 1012-1013, 2008.

[62] J. J. Vredenburgh, A. Desjardins, J. E. Herndon II et al., "Phase II trial of bevacizumab and irinotecan in recurrent malignant glioma," Clinical Cancer Research, vol. 13, no. 4, pp. 1253-1259, 2007.

[63] A. Lai, E. Filka, B. McGibbon et al., "Phase II pilot study of bevacizumab in combination with temozolomide and regional radiation therapy for up-front treatment of patients with newly diagnosed glioblastoma multiforme: interim analysis of safety and tolerability," International Journal of Radiation Oncology, Biology, Physics, vol. 71, no. 5, pp. 1372-1380, 2008.

[64] S. Gururangan, S. N. Chi, T. Y. Poussaint et al., "Lack of efficacy of bevacizumab plus irinotecan in children with recurrent malignant glioma and diffuse brainstem glioma: a pediatric brain tumor consortium study," Journal of Clinical Oncology, vol. 28, no. 18, pp. 3069-3075, 2010.

[65] A. Narayana, S. Kunnakkat, J. Chacko-Mathew et al., "Bevacizumab in recurrent high-grade pediatric gliomas," NeuroOncology, vol. 12, no. 9, pp. 985-990, 2010.

[66] C. Parekh, R. Jubran, A. Erdreich-Epstein et al., “Treatment of children with recurrent high grade gliomas with a bevacizumab containing regimen," Journal of Neuro-Oncology, vol. 103, no. 3, pp. 673-680, 2011.

[67] I. F. Pollack, S. D. Finkelstein, J. Burnham et al., "Age and TP53 mutation frequency in childhood malignant gliomas: results in a multi-institutional cohort," Cancer Research, vol. 61, no. 20, pp. 7404-7407, 2001.

[68] I. F. Pollack, S. D. Finkelstein, J. Woods et al., "Expression of p53 and prognosis in children with malignant gliomas," The New England Journal of Medicine, vol. 346, no. 6, pp. 420-427, 2002.

[69] J. A. Simon and R. E. Kingston, "Mechanisms of polycomb gene silencing: knowns and unknowns," Nature Reviews Molecular Cell Biology, vol. 10, no. 10, pp. 697-708, 2009.

[70] G. Wu, A. Broniscer, T. A. McEachron et al., "Somatic histone $\mathrm{H} 3$ alterations in pediatric diffuse intrinsic pontine gliomas and non-brainstem glioblastomas," Nature Genetics, vol. 44, no. 3, pp. 251-253, 2012.

[71] D. Sturm, H. Witt, V. Hovestadt et al., "Hotspot mutations in H3F3A and IDH1 define distinct epigenetic and biological subgroups of glioblastoma," Cancer Cell, vol. 22, no. 4, pp. 425437, 2012.

[72] D.-A. Khuong-Quang, P. Buczkowicz, P. Rakopoulos et al., "K27M mutation in histone H3.3 defines clinically and biologically distinct subgroups of pediatric diffuse intrinsic pontine gliomas," Acta Neuropathologica, vol. 124, no. 3, pp. 439-447, 2012.

[73] G. H. Gielen, M. Gessi, J. Hammes, C. M. Kramm, A. Waha, and T. Pietsch, "H3F3A K27M mutation in pediatric CNS tumors: a marker for diffuse high-grade astrocytomas," The American Journal of Clinical Pathology, vol. 139, no. 3, pp. 345-349, 2013.

[74] A. Dhayalan, R. Tamas, I. Bock et al., "The ATRX-ADD domain binds to $\mathrm{H} 3$ tail peptides and reads the combined methylation state of K4 and K9," Human Molecular Genetics, vol. 20, no. 11, pp. 2195-2203, 2011.

[75] C. M. Heaphy, R. F. de Wilde, Y. Jiao et al., "Altered telomeres in tumors with ATRX and DAXX mutations," Science, vol. 333, no. 6041, article 425, 2011.

[76] B. Wiestler, D. Capper, T. Holland-Letz et al., "ATRX loss refines the classification of anaplastic gliomas and identifies a subgroup of IDH mutant astrocytic tumors with better prognosis," Acta Neuropathologica, vol. 126, no. 3, pp. 443-451, 2013.

[77] S. Zhao, Y. Lin, W. Xu et al., "Glioma-derived mutations in IDH1 dominantly inhibit IDH1 catalytic activity and induce HIF-1 $\alpha$," Science, vol. 324, no. 5924, pp. 261-265, 2009.

[78] S. Nobusawa, T. Watanabe, P. Kleihues, and H. Ohgaki, "IDH1 mutations as molecular signature and predictive factor of secondary glioblastomas," Clinical Cancer Research, vol. 15, no. 19, pp. 6002-6007, 2009.

[79] J. Balss, J. Meyer, W. Mueller, A. Korshunov, C. Hartmann, and A. von Deimling, "Analysis of the IDH1 codon 132 mutation in 
brain tumors," Acta Neuropathologica, vol. 116, no. 6, pp. 597602, 2008.

[80] H. Yan, D. W. Parsons, G. Jin et al., "IDH1 and IDH2 mutations in gliomas," The New England Journal of Medicine, vol. 360, no. 8, pp. 765-773, 2009.

[81] M. Antonelli, F. R. Buttarelli, A. Arcella et al., "Prognostic significance of histological grading, p53 status, YKL-40 expression, and IDH1 mutations in pediatric high-grade gliomas," Journal of Neuro-Oncology, vol. 99, no. 2, pp. 209-215, 2010.

[82] C. Hartmann, J. Meyer, J. Balss et al., "Type and frequency of IDH1 and IDH2 mutations are related to astrocytic and oligodendroglial differentiation and age: a study of 1,010 diffuse gliomas," Acta Neuropathologica, vol. 118, no. 4, pp. 469-474, 2009.

[83] I. F. Pollack, R. L. Hamilton, R. W. Sobol et al., "IDH1 mutations are common in malignant gliomas arising in adolescents: a report from the Children's Oncology Group," Child's Nervous System, vol. 27, no. 1, pp. 87-94, 2011. 


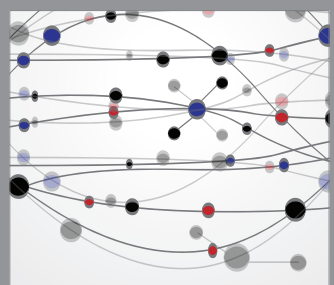

The Scientific World Journal
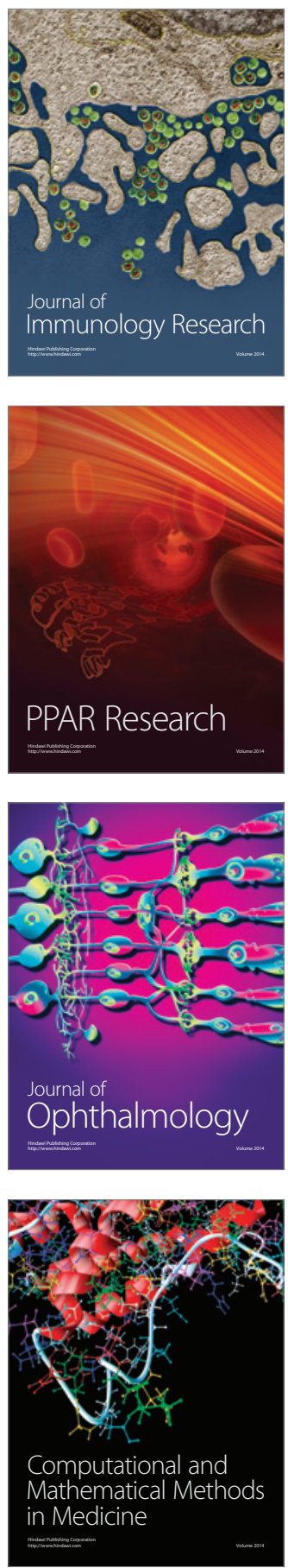

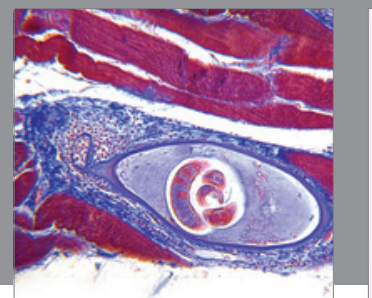

Gastroenterology

Research and Practice
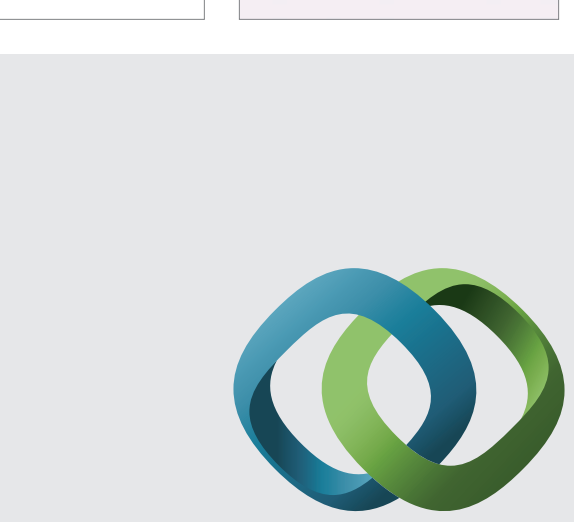

\section{Hindawi}

Submit your manuscripts at

http://www.hindawi.com
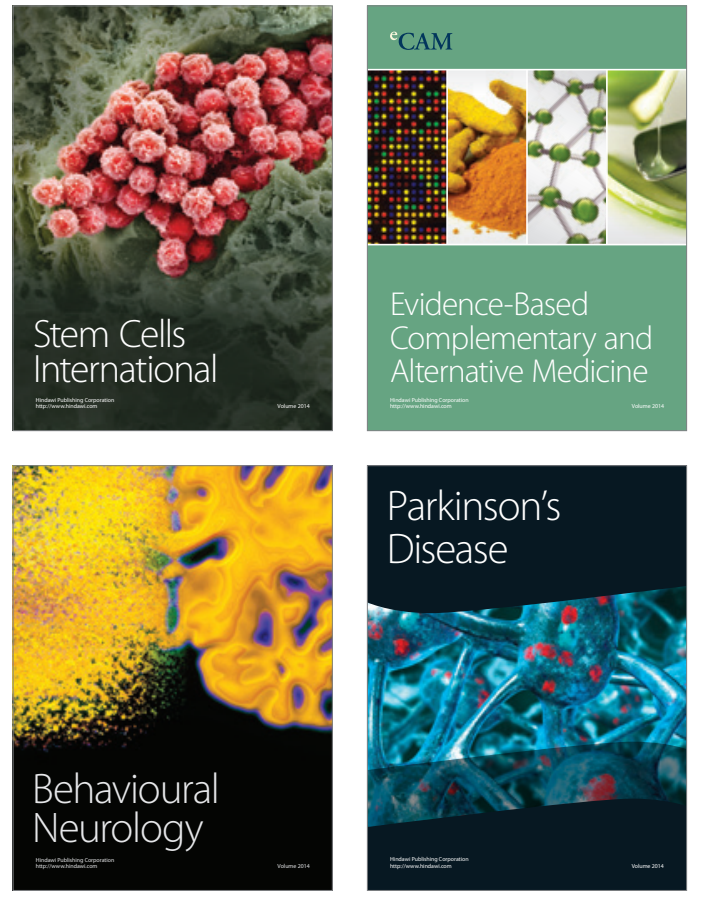
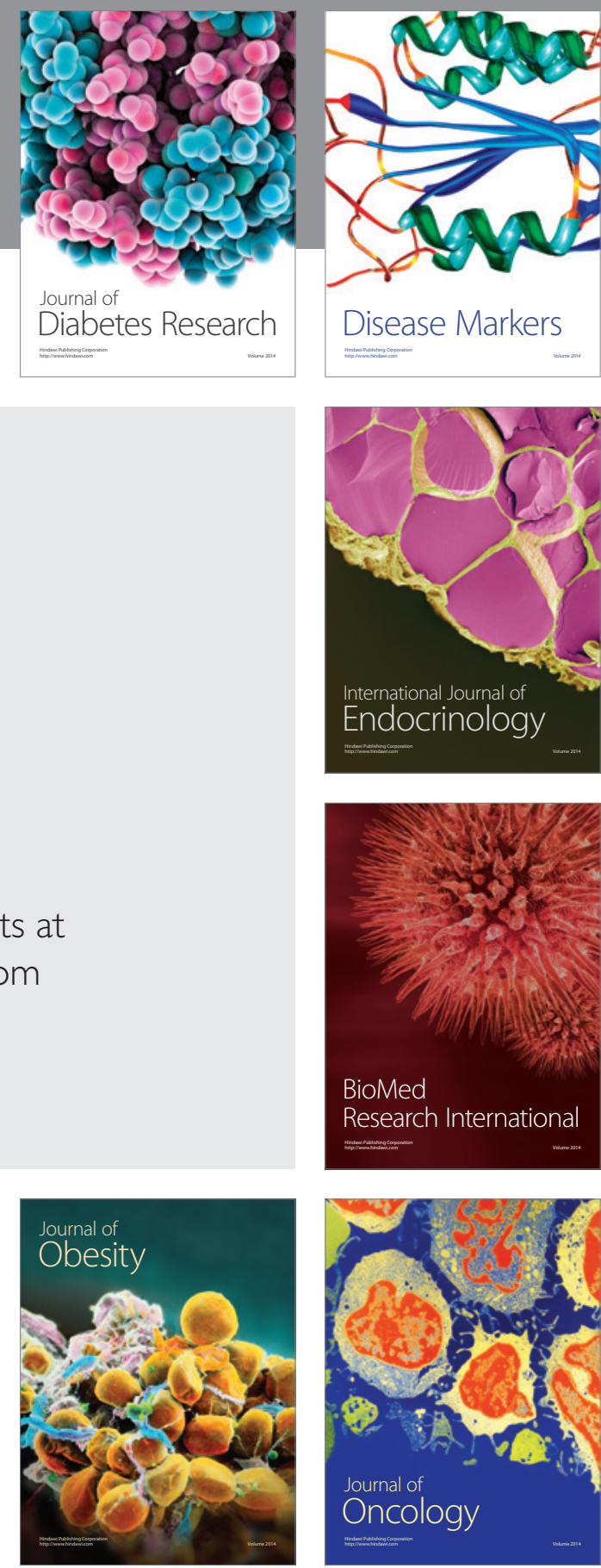

Disease Markers
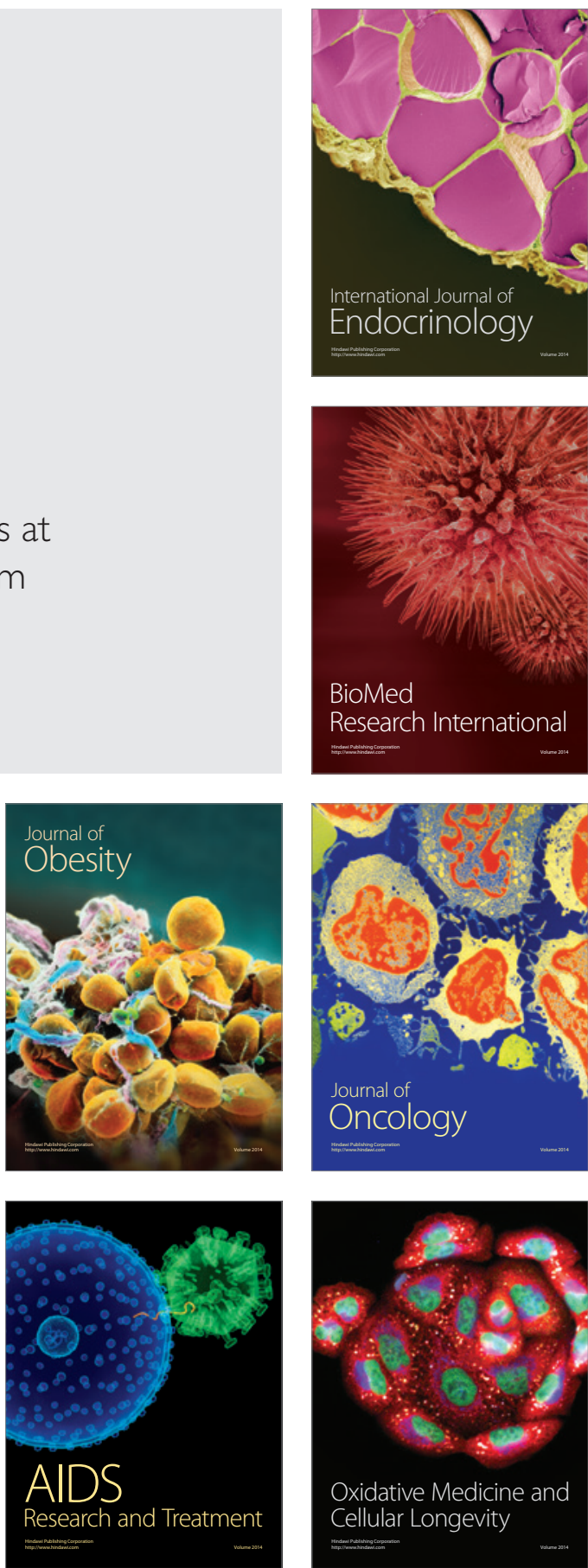